\title{
Conhecimento dos acadêmicos e profissionais da fisioterapia sobre dor: uma revisão sistemática
}

\section{Knowledge of physical therapy students and professionals about pain: a systematic review}

\author{
Alan Carlos Nery dos Santos ${ }^{1,2}$, Monique Lima Barbosa ${ }^{1}$, Anelize Gimenez de Souza ${ }^{1}$, Jefferson Petto ${ }^{1,2}$ \\ ${ }^{1}$ Grupos de Pesquisa Ciências da Saúde em Fisioterapia, Universidade Salvador (UNIFACS) - Feira de Santana (BA), Brasil. \\ ${ }^{2}$ Curso de Fisioterapia, Faculdade Adventista da Bahia (FADBA) - Cachoeira (BA), Brasil.
}

http://dx.doi.org/10.7322/abcshs.v42i2.1010

\begin{abstract}
RESUMO
Estudos demonstram que acadêmicos e profissionais da fisioterapia apresentam limitações no conhecimento sobre diversos aspectos do tema "dor". Isso pode implicar em abordagens inadequadas e em maior sofrimento dos pacientes. Assim, objetivou-se revisar estudos que investigaram o conhecimento de acadêmicos e profissionais da fisioterapia sobre dor, bem como os que propuseram estratégias para modificar tal conhecimento. Trata-se de uma revisão sistemática, e foram consultadas as bases de dados EBSCOhost, LILACS, PubMed, REDALYC e SciELO, por meio do cruzamento das palavras-chave: "pain", "knowledge", "education", "curriculum", "physical therapy specialty" e os seus correspondentes em português. Foram considerados elegíveis os estudos observacionais ou experimentais que analisaram o conhecimento sobre aspectos clínicos, fisiopatológicos e manejo da dor. Também foram elegíveis os que testaram estratégias para modificar o conhecimento sobre dor. A triagem pelos títulos e resumo resultou em 84 estudos. Contudo, apenas cinco foram incluídos. Esses analisaram o conhecimento sobre aspectos gerais da dor, sendo publicados entre 2003 e 2016. Apenas $60 \%$ dos estudos investigaram participantes da fisioterapia, sendo que $100 \%$ deles verificaram o conhecimento por meio de questionários autoaplicáveis. Quatro estudos utilizaram questionários validados. Somente $20 \%$ testaram estratégias para modificar o conhecimento sobre dor. Os resultados desta revisão indicam que o tema "dor" é abordado apenas como subtema complementar, o que, embora permita o aprendizado, não tem sido suficiente para garanti-lo. Profissionais da fisioterapia apresentam inconsistência entre o conhecimento teórico e os seus papeis no manuseio e na assistência humanizada da dor. Estratégias de ensino-aprendizagem ativas podem aprimorar o conhecimento acadêmico sobre dor.
\end{abstract}

Palavras-chave: fisioterapia; dor; conhecimento; educação; currículo.

\section{ABSTRACT}

Studies show that physical therapy students and professionals have limited knowledge about various aspects of the subject "pain". This may result in inadequate approach and increases the patients' suffering. Thus, it was aimed to review studies that investigated the physiotherapy students' and professionals' knowledge about pain, as well as to propose strategies for changing it. This is a systematic review in which EBSCOhost, LILACS, PubMed, REDALYC and SciELO databases were consulted by crossing the following keywords and its matching terms in Portuguese: "pain", "knowledge", "education", "curriculum", "physical therapy specialty". Crosssectional or experimental studies addressing knowledge about pain clinical and pathophysiological aspects, and management were eligible. Studies that tested strategies to modify knowledge about pain were also qualified. Screening by title and abstract resulted in 84 studies. However, only five studies were included, which analyzed the knowledge about general aspects of pain and were published between 2003 and 2016 . Only $60 \%$ of studies investigated volunteers from the field of physical therapy, whereas $100 \%$ of them had their knowledge verified in self-reported questionnaires. Four studies used validated questionnaires. Only 20\% of them tested strategies to modify the knowledge about pain. The results of this review are that the topic "pain" is addressed as sub-topic only. Although it allows learning, it is not enough to ensure it. Physical therapy professionals are inconsistent as to theoretical knowledge and practice on pain management and human assistance. Teaching and learning strategies may improve students' knowledge about pain.

Keywords: physical therapy specialty; pain; knowledge; education; curriculum.

Recebido em: 27/03/2016

Revisado em: 31/05/2016

Aprovado em: 30/06/2016

Autor para correspondência: Alan Carlos Nery dos Santos - Universidade Salvador (UNIFACS), Campus Santa Mônica - Rua Rio Tinto, 152 - Santa Mônica CEP: 4077-140 - Feira de Santana (BA), Brasil - E-mail: allannery.santos@hotmail.com

Conflito de interesses: nada a declarar 


\section{INTRODUÇÃO}

Estudos científicos nacionais e internacionais apontam que acadêmicos e profissionais da saúde apresentam deficit no conhecimento sobre mecanismos fisiopatológicos, avaliação e tratamento da dor ${ }^{1-3}$. Esses achados podem refletir os resultados negativos de estudos que apontaram que a dor, seja ela aguda ou crônica, é subdiagnosticada, mal avaliada, subtratada, com baixa resolutividade e por vezes negligenciada em vários níveis de atenção à saúde ${ }^{4-6}$.

Por outro lado, chama atenção que há mais de uma década a dor passou a ser considerada como o quinto sinal vital; assim, sua adequada avaliação e controle são direito do paciente e dever das equipes de saúde ${ }^{7}$. Outro ponto relevante é que a dor é um importante problema de saúde pública mundial, caracterizada como um fenômeno multidimensional, diretamente influenciado por fatores individuais, culturais, sociais, psicológicos, ambientais e físicos. Sendo também, segundo a Associação Internacional para o Estudo da Dor (em inglês, International Association for the Study of Pain), uma experiência sensorial e emocional desagradável, associada a lesões reais ou potenciais ${ }^{8}$.

Portanto, com base no raciocínio apresentado e no fato de que o fenômeno em questão é de elevada complexidade, com impactos biopsicossociais desastrosos, além do elevado custo financeiro aos cofres públicos e privados, fica evidente a necessidade real $\mathrm{e}$ atual de que os acadêmicos e profissionais de saúde, incluindo a fisioterapia, estejam adequadamente habilitados para realizar ações de prevenção, avaliação e tratamento da dor em todas as esferas de atenção à saúde ${ }^{8-10}$. Vale ressaltar que mais da metade dos pacientes que procuram o serviço de fisioterapia apresentam como sintoma principal a dor, a qual tem impactos diretos sobre a funcionalidade durante as atividades de vida diária, laborais e qualidade de vida ${ }^{10-12}$.

Evidências também apontam que a fisioterapia é um recurso não medicamentoso eficiente para o tratamento da dor e das alterações funcionais decorrentes desta. De fato, vários mecanismos e efeitos podem ser influenciados pelas técnicas físicas e cognitivas comportamentais da fisioterapia no tratamento do paciente com dor $^{13}$. Entretanto, a seleção das abordagens mais adequadas parece dependente de alto grau de conhecimento técnico, aliado à melhor evidência científica ${ }^{13,14}$. Assim, devido à carência de estudos sobre o nível de conhecimento dos acadêmicos e profissionais da saúde sobre a dor e suas abordagens terapêuticas, este estudo objetivou revisar as pesquisas que investigaram o conhecimento dos acadêmicos e profissionais da fisioterapia sobre dor, bem como as publicações que propuseram estratégias para modificar tal conhecimento.

\section{MÉTODOS}

\section{Tipo de pesquisa}

Trata-se de uma revisão sistemática de estudos originais que objetivaram analisar o conhecimento dos acadêmicos e profissionais da área da fisioterapia sobre a dor e suas abordagens terapêuticas, bem como os que testaram estratégias de ensino-aprendizagem e educação continuada capazes de modificar tal conhecimento.

\section{Estratégia de busca}

A busca por potenciais estudos foi desenvolvida por dois autores independentes, no período de dezembro de 2015 a março de 2016. Foram consultadas as bases de dados eletrônicas: EBSCOhost, LILACS, PubMed, REDALYC e SciELO. Para a seleção dos manuscritos, utilizou-se como descritores as seguintes palavras-chave: "pain", "knowledge", "education", "curriculum", "physical therapy specialty", e os seus correspondentes em português. Essas foram cruzadas da seguinte forma: "pain AND knowledge AND physical therapy specialty"; "pain AND education AND physical therapy specialty"; e "pain AND curriculum AND physical therapy specialty".

\section{Critérios de elegibilidade e exclusão}

Adotou-se como critério de elegibilidade: estudos originais com delineamento observacional ou experimental que analisaram o conhecimento de acadêmicos ou profissionais da área da fisioterapia sobre aspectos gerais da dor, como fisiopatologia, métodos de avaliação e abordagens terapêuticas da dor; e estudos que aplicaram estratégias de ensino-aprendizagem ou educação continuada para aprimorar o conhecimento de acadêmicos ou de profissionais de nível superior (da área da fisioterapia) sobre a dor. Para inclusão, os estudos deveriam estar disponíveis na íntegra, publicados em língua portuguesa ou inglesa, até março de 2016. Foram adotados como critérios de exclusão os artigos: publicados em periódicos não indexados; que não descreveram a metodologia de avaliação do conhecimento sobre dor; que não descreveram o quantitativo de participantes da área da fisioterapia; e de revisão.

\section{Seleção dos estudos e extração de dados}

O somatório de artigos recuperados nas bases de dados foi inicialmente triado por meio da leitura dos títulos. Em seguida, foram excluídos os estudos em duplicidade. Na sequência, realizou-se nova leitura dos títulos e resumos para verificar se atendiam aos critérios de elegibilidade adotados neste estudo. Conforme mostra o Quadro 1, os que se adequaram aos critérios de seleção foram recuperados para leitura do texto completo, nova avaliação quanto aos critérios de elegibilidade e extração dos dados referentes a:

- autor e ano de publicação;

- objetivo;

- população;

- métodos (instrumento de avaliação do conhecimento, estratégias de educação continuada, de ensino-aprendizagem); e

- principais conclusões.

Por fim, as referências dos estudos incluídos nesta revisão foram analisadas com o objetivo de verificar a existência de artigos elegíveis não identificados nas buscas nas bases de dados selecionadas. 
A Figura 1 sintetiza o processo de seleção dos estudos que compõe o escopo desta revisão sistemática.

\section{RESULTADOS}

As pesquisas nas bases de dados selecionadas resultaram na identificação de 84 referências, as quais foram inicialmente triadas pelos títulos e resumos. Contudo, 28 artigos foram excluídos por serem duplicados e 51 por não estarem de acordo com os critérios de seleção adotados. Portanto, apenas cinco estudos foram elegíveis para fazer parte do escopo desta revisão sistemáti$\mathrm{ca}^{2,3,15-17}$. Nenhum artigo foi encontrado pela busca manual na lista de referências dos estudos selecionados.

Com base nos estudos incluídos, um total de 129 acadêmicos e 217 profissionais da área da fisioterapia foram avaliados. Todos os estudos revisados analisaram o conhecimento sobre aspectos

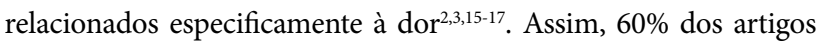
investigaram o conhecimento de acadêmicos do primeiro ao último período do curso de fisioterapia, de instituições de ensino superior públicas e privadas ${ }^{2,15}$. Apenas $40 \%$ dos estudos avaliaram o conhecimento de profissionais. O primeiro ${ }^{2}$ avaliou uma amostra probabilística composta por 207 fisioterapeutas da África do Sul, os quais apresentaram uma média de 14 anos de experiência clínica em fisioterapia e 12 anos no tratamento de atletas. Já o segundo ${ }^{16}$ foi composto por voluntários graduados em fisioterapia há pelo menos um ano, e que exerciam suas atividades profissionais em um hospital universitário federal na cidade de Aracajú - SE.

Em geral, a análise do conhecimento dos acadêmicos foi baseada em questionários autoaplicáveis contendo entre 12 e 27 questões objetivas, sendo que $40 \%$ dos estudos adicionaram ou removeram perguntas do questionário original. Por outro lado, um estudo ${ }^{2}$ com profissionais e todos os estudos ${ }^{3,15,17}$ com acadêmicos utilizaram ferramentas previamente validadas. Essas continham questões destinadas a caracterizar a amostra, avaliar os aspectos gerais e terapêuticos da dor, além de conhecer as características neurofisiológicas, psicológicas, desenvolvimento, avaliação/ medição, farmacologia, e aspectos cognitivos/comportamentais do conhecimento da dor. Apenas um estudo ${ }^{17}$, com acadêmicos, verificou o conhecimento e aplicou intervenções para aprimorar o conhecimento da dor. Os estudos com profissionais somente sugeriram a necessidade de instituir políticas de educação continuada para melhorar o desempenho técnico e científico dos $\operatorname{avaliados}^{2,16}$.

Os principais achados dos estudos revisados indicam que o tema "dor" não é abordado como assunto principal nas disciplinas das escolas onde as pesquisas foram desenvolvidas. Ainda assim, foi mostrado que os acadêmicos de fisioterapia apresentam conhecimento adequado quanto à fisiologia e à avaliação da dor. Entretanto, nos aspectos relacionados às terapêuticas gerais e aos tratamentos farmacológicos e não farmacológico da dor, o conhecimento foi inadequado ${ }^{3,15,17}$. Entre os profissionais foi observada inconsistência entre o conhecimento teórico e seus papeis no manuseio e assistência humanizada da dor ${ }^{16}$, assim como conhecimento insuficiente para garantiradequadas avaliação, mensuração e gestão da dor ${ }^{2}$. O único estudo ${ }^{17}$ que interviu sobre o conhecimento identificou que estratégias de ensino-aprendizagem ativas, aplicadas em uma disciplina específica para o tema (dor), foi suficiente para aprimorar o conhecimento sobre neurofisiologia da dor. Por fim, o Quadro 1 apresenta a síntese qualitativa dos estudos originais que compõem esta revisão.

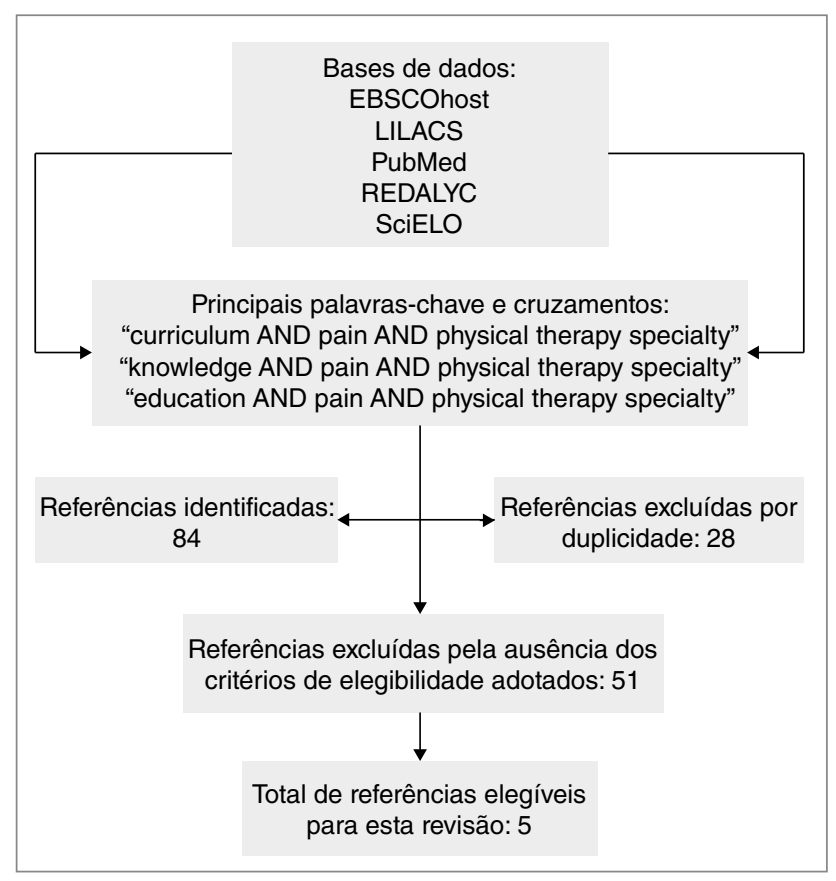

Figura 1: Fluxograma de seleção dos estudos

Quadro 1: Síntese qualitativa dos estudos que analisaram os conhecimentos de acadêmicos e profissionais da fisioterapia sobre dor e suas abordagens

\begin{tabular}{|c|c|c|c|c|}
\hline Autor & Objetivo & População & Método & Principais conclusões \\
\hline $\begin{array}{l}\text { Sereza e } \\
\text { Dellaroza } \\
(2003)^{3}\end{array}$ & $\begin{array}{l}\text { Identificar as disciplinas } \\
\text { e conteúdos sobre o } \\
\text { tema dor, identificar os } \\
\text { conceitos adquiridos } \\
\text { pelos alunos referentes } \\
\text { aos aspectos gerais } \\
\text { da dor, à avaliação da } \\
\text { dor e às terapêuticas } \\
\text { analgésicas. }\end{array}$ & $\begin{array}{l}170 \text { acadêmicos } \\
\text { da área da } \\
\text { saúde, sendo } \\
30 \text { estudantes } \\
\text { do último ano } \\
\text { do curso de } \\
\text { Fisioterapia. }\end{array}$ & $\begin{array}{l}\text { Questionário autoaplicável formado } \\
\text { por } 27 \text { questões objetivas, sendo } 12 \\
\text { sobre aspectos gerais da dor e } 15 \\
\text { sobre terapêuticas da dor. }\end{array}$ & $\begin{array}{l}\text { O tema dor é abordado na graduação } \\
\text { como subtema complementar em diversas } \\
\text { disciplinas. } \\
\text { Os alunos demonstraram adequados } \\
\text { conhecimentos sobre a fisiologia e a } \\
\text { avaliação da dor, contudo demonstraram } \\
\text { fragilidades em conceitos referentes a } \\
\text { terapêuticas. }\end{array}$ \\
\hline
\end{tabular}


Quadro 1: Continuação

\begin{tabular}{|c|c|c|c|c|}
\hline Autor & Objetivo & População & Método & Principais conclusões \\
\hline $\begin{array}{l}\text { Alves et al. } \\
(2013)^{15}\end{array}$ & $\begin{array}{l}\text { Mensurar o } \\
\text { conhecimento sobre } \\
\text { dor e terapêuticas pelos } \\
\text { acadêmicos do curso de } \\
\text { fisioterapia em centro } \\
\text { universitário, elucidando } \\
\text { as abordagens } \\
\text { terapêuticas de maior } \\
\text { conhecimento para } \\
\text { o controle da dor, } \\
\text { determinando a evolução } \\
\text { do conhecimento sobre o } \\
\text { tema no decorrer } \\
\text { do curso. }\end{array}$ & $\begin{array}{l}85 \text { acadêmicos } \\
\text { do } 1^{\circ} \text { ao } 10^{\circ} \\
\text { período do curso } \\
\text { de Fisioterapia. }\end{array}$ & $\begin{array}{l}\text { Questionário autoaplicável proposto } \\
\text { e validado por Sereza e Dellaroza }{ }^{3} \text {, } \\
\text { formado por } 27 \text { questões objetivas, } \\
\text { sendo } 12 \text { sobre aspectos gerais da } \\
\text { dor e } 15 \text { sobre terapêuticas da dor. } \\
\text { Os pesquisadores acresceram cinco } \\
\text { questões sobre fisioterapia no controle } \\
\text { da dor ao questionário original. }\end{array}$ & $\begin{array}{l}\text { O tema dor não é abordado como assunto } \\
\text { principal e sim como complementar em } \\
\text { várias disciplinas. } \\
\text { Os alunos mostraram maior conhecimento } \\
\text { em áreas como fisiopatologia, subjetividade } \\
\text { e avaliação, tratamento não farmacológico } \\
\text { e fisioterapia no quadro álgico. } \\
\text { Em aspectos como terapêuticas gerais da } \\
\text { dor e tratamento farmacológico com uso de } \\
\text { opioides, todos os grupos demonstraram } \\
\text { percentagens de acertos abaixo de } 50 \% \text {. }\end{array}$ \\
\hline $\begin{array}{l}\text { Clenzos et al. } \\
(2013)^{2}\end{array}$ & $\begin{array}{l}\text { Fornecer informações } \\
\text { em relação ao } \\
\text { conhecimento sobre dor } \\
\text { entre fisioterapeutas } \\
\text { desportistas e } \\
\text { fisioterapeutas } \\
\text { especialistas em } \\
\text { manipulações } \\
\text { ortopédicas. }\end{array}$ & $\begin{array}{l}207 \\
\text { fisioterapeutas } \\
\text { graduados, } \\
\text { sendo } 119 \\
\text { pós-graduados, } \\
33 \text { mestres e } 2 \\
\text { pós-doutores. } \\
\text { Um total de } 191 \\
\text { participantes } \\
\text { tinham } \\
\text { experiência } \\
\text { clínica, em média } \\
15 \text { anos; } 119 \\
\text { participantes } \\
\text { tinham } \\
\text { experiência com } \\
\text { atletas. }\end{array}$ & $\begin{array}{l}\text { Avaliados pelo PKAQ, instrumento } \\
\text { apropriado para profissionais da } \\
\text { saúde. O questionário abrange } \\
\text { questões fisiológicas, psicológicas, } \\
\text { desenvolvimento, avaliação/ } \\
\text { medição, farmacologia e aspectos } \\
\text { cognitivos/comportamentais do } \\
\text { conhecimento da dor. } \\
\text { A seção sobre farmacologia foi excluída } \\
\text { do estudo visto que não compõe o } \\
\text { escopo de atuação dos profissionais de } \\
\text { fisioterapia da África do Sul. }\end{array}$ & $\begin{array}{l}\text { Os profissionais de fisioterapia, desportistas } \\
\text { e ortopédicos manipulativos podem ter } \\
\text { conhecimento inadequado sobre dor. } \\
\text { Com base em uma pontuação mínima } \\
\text { de } 75 \% \text { no PKAQ, foi constatado que os } \\
\text { profissionais poderiam ter conhecimento } \\
\text { adequado para avaliar e tratar a dor. } \\
\text { Também foi detectada limitação no } \\
\text { conhecimento para avaliação e mensuração } \\
\text { da dor, bem como dos processos } \\
\text { envolvidos no surgimento desta. Em } \\
\text { geral, foi mostrado que os profissionais } \\
\text { de fisioterapia poderiam ter conhecimento } \\
\text { insuficiente para garantir adequada gestão } \\
\text { e avaliação da dor. }\end{array}$ \\
\hline $\begin{array}{l}\text { Ribeiro et al. } \\
(2015)^{16}\end{array}$ & $\begin{array}{l}\text { Descrever o } \\
\text { conhecimento dos } \\
\text { profissionais de uma } \\
\text { equipe hospitalar } \\
\text { multidisciplinar sobre o } \\
\text { tema dor e analgesia. }\end{array}$ & $\begin{array}{l}82 \text { profissionais } \\
\text { da saúde, sendo } \\
10 \text { fisioterapeutas } \\
\text { formados há mais } \\
\text { de } 1 \text { ano. }\end{array}$ & $\begin{array}{l}\text { Questionário autoaplicável com } \\
\text { questões relacionadas a dados } \\
\text { sociodemográficos, acadêmicos } \\
\text { e profissionais; conceitos } \\
\text { básicos sobre dor; avaliação e } \\
\text { mensuração do fenômeno doloroso; } \\
\text { tratamentos farmacológicos e não } \\
\text { farmacológicos para o alívio da dor; } \\
\text { aquisição do conhecimento sobre } \\
\text { dor e analgesia. }\end{array}$ & $\begin{array}{l}\text { Existe inconsistência entre o embasamento } \\
\text { teórico dos participantes da pesquisa e } \\
\text { seus papéis no manuseio da dor e na } \\
\text { assistência humanizada. } \\
\text { Os profissionais afirmaram ter adquirido } \\
\text { informações sobre avaliação e manuseio } \\
\text { da dor durante a graduação, embora } \\
\text { muitos considerem que a experiência } \\
\text { profissional conferiu maior incremento } \\
\text { a tal conhecimento. Esse fator pode ser } \\
\text { prejudicial à assistência, ao passo que as } \\
\text { práticas baseadas apenas em experiências } \\
\text { pode perpetuar conceitos equivocados } \\
\text { sobre o tema. } \\
\text { Sugere a necessidade de uma comissão } \\
\text { especializada em dor, elaboração de } \\
\text { protocolos sistemáticos de avaliação e } \\
\text { educação continuada sobre dor. }\end{array}$ \\
\hline $\begin{array}{l}\text { Marques } \\
\text { et al. }(2016)^{17}\end{array}$ & $\begin{array}{l}\text { Avaliar o grau do } \\
\text { conhecimento } \\
\text { neurofisiológico da } \\
\text { dor de estudantes de } \\
\text { fisioterapia, após serem } \\
\text { utilizadas estratégias } \\
\text { ativas de ensino- } \\
\text { aprendizagem na } \\
\text { abordagem do tema. }\end{array}$ & $\begin{array}{l}14 \text { acadêmicos } \\
\text { do } 5^{\circ} \text { período } \\
\text { do curo de } \\
\text { Fisioterapia. }\end{array}$ & $\begin{array}{l}\text { Questionário Neurofisiológico da } \\
\text { Dor, instrumento autoaplicável, } \\
\text { originalmente desenvolvido com } 19 \\
\text { itens que avaliam o conhecimento } \\
\text { relacionado à neurofisiologia da } \\
\text { dor, no qual cada item apresenta } \\
3 \text { opções de resposta: verdadeiro, } \\
\text { falso e indeciso. Após a avaliação } \\
\text { das propriedades psicométricas, } \\
\text { foi constatado que apenas } 12 \text { itens } \\
\text { são necessários para alcançar os } \\
\text { mesmos resultados do questionário } \\
\text { original. } \\
\text { Escores iguais ou superiores a } 65 \% \\
\text { de acerto em } 90 \% \text { dos participantes } \\
\text { foram considerados satisfatórios para } \\
\text { avaliar a aquisição do conhecimento } \\
\text { neurofisiológico da dor. }\end{array}$ & $\begin{array}{l}\text { Na avaliação inicial os acadêmicos } \\
\text { obtiveram um percentual de } 65 \% \text { de } \\
\text { acertos, o que foi considerado insatisfatório. } \\
\text { O grau de conhecimento neurofisiológico } \\
\text { da dor de estudantes de Fisioterapia } \\
\text { foi aperfeiçoado pela realização de } \\
\text { disciplinas que abordam de forma } \\
\text { específica conteúdos sobre a dor, tendo } \\
\text { os acadêmicos atingido } 90 \% \text { de acertos } \\
\text { na avaliação final. As estratégias ativas de } \\
\text { ensino-aprendizagem demonstraram ser } \\
\text { capazes de favorecer essa construção de } \\
\text { conhecimento. }\end{array}$ \\
\hline
\end{tabular}




\section{DISCUSSÃO}

Embora seja aceito pela comunidade acadêmica e científica que a dor é um importante sinal vital, sendo também o principal sintoma presente em mais da metade dos pacientes que procuram o serviço de fisioterapia ${ }^{10,11}$, parece, a priori, que as instituições de ensino superior estão tendo dificuldade para desenvolver uma grade curricular mínima sobre o assunto em questão ${ }^{18}$. Isso porque, com base nos estudos selecionados, o tema tem sido abordado na graduação apenas como subtema complementar em diversas disciplinas, o que parece não ser suficiente para um adequado aprendizado ${ }^{3,15,17}$.

De fato, ainda que a literatura seja limitada quanto a estudos voltados ao monitoramento do conhecimento de acadêmicos e profissionais sobre temas relacionados à atuação dos fisioterapeutas, estudos apontam que o conhecimento destes pode não estar sendo suficientemente adequado para o exercício de práticas voltadas aos cuidados de pacientes, principalmente aqueles com dor ${ }^{2,3,15,16}$. Assim, foi demonstrado por Sereza e Dellaroza ${ }^{3}$ que, embora os acadêmicos demonstrem conhecimento adequado sobre fisiopatologia e avaliação da dor, há fragilidades em conceitos referentes às terapêuticas medicamentosas e não medicamentosas da dor. Esses dados são apoiados pelo estudo de Alves et al. ${ }^{15}$, que, apesar de publicado dez anos mais tarde, mostrou resultados similares aos do estudo anteriormente citado. Vale ressaltar que no estudo em questão foi utilizado o mesmo questionário aplicado por Sereza e Dellaroza ${ }^{3}$, o que indica que mesmo após dez anos os problemas na formação acadêmica do fisioterapeuta para o manejo clínico da dor continuam os mesmos.

Por outro lado, é preocupante notar que essas limitações também foram identificadas em profissionais, os quais apresentaram tempo de formação em fisioterapia de 1 a $14 \operatorname{anos}^{2,16}$ e atuavam em centros hospitalares e desportivos. Esses dados sugerem que a prática profissional isoladamente pode não ser suficiente para aprimorar e consolidar conteúdos referentes à dor. Em concordância com essa hipótese, Ribeiro et al. ${ }^{16}$ identificaram inconsistência entre o conhecimento teórico e o papel do fisioterapeuta no manuseio e na assistência humanizada da dor. Nessa pesquisa, embora os profissionais avaliados tenham afirmado ter adquirido informações sobre abordagem da dor durante a graduação, muitos deles consideraram que a experiência profissional conferiu maior incremento a tal conhecimento. Segundo os autores, esse fator pode ser prejudicial à assistência fisioterapêutica porque a prática baseada apenas em experiências, sem respaldo em evidências científicas, pode perpetuar conceitos clínicos equivocados. Corroborando esses dados, Clenzos et al. ${ }^{2}$ afirmaram que embora a amostra de profissionais avaliados por eles tivesse atingido a pontuação mínima no questionário por eles aplicado, os mesmos poderiam ter conhecimento insuficiente para garantir a avaliação, a mensuração e a gestão adequada da dor.

Assim, fica clara a necessidade não somente de repensar a forma de abordar o tema "dor" durante a graduação, mas também de monitorar tal conhecimento após a conclusão do curso. Além disso, cabe nesse contexto a adoção de estratégias para minimizar as lacunas no conhecimento sobre esse tema. Neste aspecto, Marques et al. ${ }^{17}$ avaliaram o grau de conhecimento sobre neurofisiologia da dor em estudantes do quinto período do curso de fisioterapia por meio do questionário de neurofisiologia da dor. Os pesquisadores instituíram estratégias de ensino com base em metodologias ativas de ensino-aprendizagem. Na ocasião, foram realizados 12 encontros formativos durante 1 semestre, com a finalidade de proporcionar a construção do conhecimento sobre neurofisiologia da dor. As estratégias de ensino ativo foram: estudo de texto, dramatização, estudo dirigido e estratégias de educação em saúde. Com base na reavaliação do conhecimento, os autores perceberam que as estratégias de ensino-aprendizagem adotadas foram satisfatórias para aperfeiçoar o conhecimento sobre neurofisiologia da dor por parte dos acadêmicos avaliados, o que sugere que a construção de uma disciplina específica possa ser o ideal para a temática em questão.

Ainda são necessários estudos com segmento longitudinal para que se possa avaliar a evolução dos acadêmicos quanto ao conhecimento sobre dor, durante a formação acadêmica, e a assistência a pacientes com quadros álgicos, uma vez que os estudos com profissionais também identificaram limitações no conhecimento e influência negativa dessas sobre a capacidade de prestar assistência adequada e humanizada ao paciente com dor. Outro ponto que precisa ser melhor analisado é o quanto a regionalização dos estudos influencia nos resultados encontrados. Assim, também cabe uma análise dos currículos dos cursos de fisioterapia das diversas regiões do Brasil, uma vez que os estudos analisados demonstram a não existência de uma disciplina específica, a qual, segundo o único resultado encontrado, pode proporcionar melhor conhecimento aos acadêmicos sobre a dor.

Quanto aos profissionais, embora seja recomendada a adoção de estratégia de educação continuada para aprimorar o conhecimento sobre a dor, não foram identificados estudos que tivessem avaliado tal estratégia ${ }^{2,16}$. Em outras temáticas como a prática baseada em evidências, tem sido documentado que programas de educação continuada, assim como o tempo dedicado à leitura de artigos científicos são essenciais para o aprimoramento dos profissionais que visam a sua implantação na prática clínica. Contudo, segundo Dias e Dias ${ }^{14}$, ainda há um grande abismo entre a qualidade da formação profissional e as habilidades necessárias para desenvolver ações clínicas embasadas em evidências científicas. Assim, ainda são necessárias estratégias - tanto para o encorajamento de profissionais quanto para a formação dos acadêmicos - voltadas à promoção da prática baseada em dados científicos.

Por fim, este estudo possui algumas limitações, como as inerentes à metodologia adotada e a não avaliação da qualidade metodológica dos estudos selecionados, o que pode de alguma forma influenciar nos resultados encontrados. Isso porque apenas um dos estudos apresentou cálculo de suficiência amostral, e não foram observadas comparações entre grupos com diferentes exposições a estratégias de ensino. A ausência dessa última impossibilita inferências como as realizadas por Petto et al. ${ }^{19}$, que observaram que, além da disciplina específica, a ausência de um professor especialista no tema abordado parece influenciar negativamente no aprendizado dos acadêmicos. 


\section{CONCLUSÃO}

Esta revisão identificou que, embora a dor seja considerada o quinto sinal vital, o tema ainda é abordado em graduações de fisioterapia como subtema complementar em diversas disciplinas, o que, apesar de proporcionar aprendizado sobre mecanismos fisiopatológicos, subjetividade, avaliação e tratamento não farmacológico no quadro álgico, tem sido insuficiente para proporcionar conhecimento sobre tratamento medicamentoso com uso de opioides e estratégias fisioterapêuticas adequadas no tratamento clínico da dor.

Outro ponto preocupante é que, ainda que seja um direito das pessoas com dor, ter acesso à avaliação e ao tratamento por profissionais de saúde com adequada formação, os estudos que compõem esta revisão identificaram que existe inconsistência entre o embasamento teórico e os papeis do fisioterapeuta no manuseio e na assistência humanizada da dor. Também foi visto que esses profissionais podem apresentar conhecimento insuficiente para garantir a continuidade da gestão, avaliação, mensuração e controle da dor.

Por fim, estratégias de ensino-aprendizagem ativas com estudo de texto, dramatização, estudo dirigido e educação em saúde, introduzidas em uma disciplina com conteúdo específico para o tema dor, parecem ser suficientes para aperfeiçoar o conhecimento de acadêmicos de fisioterapia sobre dor. Por outro lado, cabe ressaltar a necessidade de revisão do currículo das escolas responsáveis pela formação dos fisioterapeutas, tendo em vista que, apesar da aparente necessidade, os centros de formação acadêmica até aqui pesquisados não possuem disciplinas específicas para o tema em questão.

\section{REFERÊNCIAS}

1. Capellini VK, Daré MF, Castral TC, Christoffel MM, Leite AM, Scochi CGS. Conhecimento e atitudes de profissionais de saúde sobre avaliação e manejo da dor neonatal. Rev Eletr Enf. 2014;16(2):361-9. http://dx.doi.org/10.5216/ree.v16i2.23611

2. Clenzos N, Naidoo N, Parker R. Physiotherapists' knowledge of pain: a cross-sectional correlational study of members of the South African Sports and Orthopaedic Manipulative Special Interest Groups. S Afr J Sports Med. 2013;25(4):95-100 http://dx.doi.org/10.7196/SAJSM.502

3. Sereza TW, Dellaroza MSG. O que está sendo aprendido a respeito da dor na UEL? Semina Cienc Biol Saúde. 2003;24:55-66. http://dx.doi.org/10.5433/1679-0367.2003v24n1p55

4. Barreto RF, Gomes CZL, Silva RM, Signorelli AAF, Oliveira LF, Cavellani CL, et al. Avaliação de dor e do perfil epidemiológico, de pacientes atendidos no pronto-socorro de um hospital universitário. Rev Dor. 2012;13(3):213-9. http://dx.doi.org/10.1590/S1806-00132012000300004

5. Marinangeli F, Narducci C, Ursini ML, Paladini A, Pasqualucci A, Gatti A, et al. Acute pain and availability of analgesia in the prehospital emergency setting in Italy: a problem to be solved. Pain Pract. 2009;9(4):282-8. http://dx.doi.org/10.1111/j.1533-2500.2009.00277.x

6. Calil AM, Pimenta CAM, Birolini D. The "oligoanalgesia problem" in the emergency care. Clinics. 2007;62(5):591-8. http://dx.doi.org/10.1590/S1807-59322007000500010

7. Luppen LS, Sampaio FH, Standñik CMB. Satisfação dos pacientes com a implantação do conceito dor o quinto sinal vital, no controle da dor pós-operatória. Rev Dor. 2011;12(1):29-34. http://dx.doi.org/10.1590/S1806-00132011000100007

8. Marquez JO. A dor e os seus aspectos multidimensionais. Cienc Cult. 2011;63(2):28-32.

9. Vlainich R, Zucchi P, Issy AM, Sakata RK. Avaliação do custo do medicamento para tratamento ambulatorial de pacientes com dor crônica. Rev Bras Anestesiol. 2010;60(4):399-405. http://dx.doi.org/10.1590/S0034-70942010000400007
10. Silva GG, Sirena SA. Perfil de encaminhamentos a fisioterapia por um serviço de Atenção Primária à Saúde, 2012. Epidemiol Serv Saúde. 2015;24(1):123-33.

http://dx.doi.org/10.5123/S1679-49742015000100014

11. Leite F, Gomes JO. Dor crônica em um ambulatório universitário de fisioterapia. Rev Ciênc Méd. 2006;15(3):211-21.

12. Mata MS, Costa FA, Souza TO, Mata ANS, Pontes JF. Dor e funcionalidade na atenção básica à saúde. Ciênc Saúde Coletiva. 2011:16(1):221-30.

http://dx.doi.org/10.1590/S1413-81232011000100025

13. Gosling AP. Mecanismos de ação e efeitos da fisioterapia no tratamento da dor. Rev Dor. 2012;13(1):65-70 http://dx.doi.org/10.1590/S1806-00132012000100012

14. Dias RC, Dias JMD. Prática baseada em evidências: uma metodologia para a boa prática fisioterapêutica. Fisioter Mov. 2006;19(1):11-6

15. Alves RC, Tavares JP, Funes RAC, Gasparetto GAR, Silva KCC Ueda TK. Evaluation of pain knowledge of Physiotherapy students from a university center. Rev Dor. 2013;14(4):272-9. http://dx.doi.org/10.1590/S1806-00132013000400008

16. Ribeiro MCO, Costa IN, Ribeiro CJN, Nunes MS, Santos B DeSantana JM. Knowledge of health professionals about pain and analgesia. Rev Dor. 2015;16(3):204-9. http://dx.doi. org/10.5935/1806-0013.20150041

17. Marques ES, Xarles T, Antunes TM, Silva KKD, Reis FJJ, Oliveira LAS, et al. Evaluation of physiologic pain knowledge by physiotherapy students. Rev Dor. 2016;17(1):29-33. http://dx.doi.org/10.5935/1806-0013.20160008

18. Castro CES, Parizotto NA, Barboza HFG. Programa mínimo sobre mecanismos de dor e analgesia para cursos de graduação em fisioterapia. Rev Bras Fisioter. 2003;7(1):85-92.

19. Petto J, Gomes VA, Oliveira FTO, Santos MPA, Barbosa PRP, Santos ACN. Importance of academic quality in the treatment of chronic venous insufficiency. Int J Cardiovasc Sci. 2016;29(1):31-6. http://www.dx.doi.org/10.5935/2359-4802.20160011 